\title{
ANÁLISE MATEMÁTICA DO MODELO DE INTERAÇÃO ENTRE A BROCA DA CANA DE AÇUCAR (DIATRAEA SACCHARALIS) E O SEU PARASITOIDE TRICHOGRAMMA GALLOI
}

\author{
Mathematical analysis of the interaction model between the sugarcane borer \\ (Diatraea Saccharalis) and its parasitoid Trichogramma Galloi \\ Maria Eduarda Ribeiro Martins, Fernando Pereira de Souza, Renato César da \\ Silva. \\ Universidade Federal de Mato Grosso do Sul - UFMS. E-mail: \\ martinsribeiroduda@gmail.com
}

RESUMO - A broca da cana de açúcar (Diatraea Saccharalis) é considerada a principal praga na cultura da cana de açúcar devido aos grandes danos à cultura causados por ela. Sua permanência pode retratar perdas significativas na produtividade e afetar a qualidade da produção de açúcar e etanol. Um dos métodos utilizados para o manejo da broca é atravésdo controle biológico mediante a inserção do parasitoide Trichogramma Galloi. A modelagem matemática auxilia na compreensão do controle biológico, pois nos permite realizar uma análise qualitativa. Neste artigo, analisamos um modelo matemático, baseado em sistemas de equações diferenciais ordinárias, de interação entre a broca da cana de açúcar, que é representada pelos estágios de ovo e larval, e seu parasitoide que é considerado em termos de ovos parasitados. Neste modelo, encontramos pontos de equilíbrio e condições para a estabilidade de cada um deles. As simulações numéricas mostram uma boa aproximação para a estabilidade.

Palavras-chave: broca da cana de açúcar; modelagem matemática; simulação numérica.

ABSTRACT - The sugarcane borer (Diatraea Saccharalis) is considered the main pest in the sugarcane crop due to the great damage it causes to the crop. Its permanence can portray significant losses in productivity and affect the quality of sugar and ethanol production. One of the methods used to manage the borer is through biological control through the insertion of the parasitoid Trichogramma Galloi. Mathematical modeling helps to understand biological control, as it allows us to carry out a qualitative analysis. In this article, we analyze a mathematical model, based on systems of ordinary differential equations, of the interaction between the sugarcane borer, which is represented by the egg and larval stages, and its parasitoid, which is considered in terms of parasitized eggs. In this model, we find equilibrium points and conditions for the stability of each one of them. Numerical simulations show a good approximation for stability.

Keywords: sugarcane borer; mathematical modeling; numerical simulation. 


\section{INTRODUÇÃO}

A produção da cana de açúcar (Saccharum Officinarum) no Brasil recebe destaque desde a época colonial, a cultura se tornou uma das mais significativas do agronegócio brasileiro por ser um dos procedimentos que mais mobilizam a economia do Brasil, fato esse que acabou conduzindo o país a se tornar o maior produtor da planta no mundo (NAVA; PINTO; SILVA, 2009). Na safra 2019/2020, a produção de cana de açúcar estimada foi de 615,98 milhões de toneladas e a área colhida estimada foi de 8,38 milhões de hectares (CONAB, 2021).

A cultura da cana é uma das mais importantes no mundo, dela obtemos dois produtos que são essenciais: o açúcar e o álcool. Além do mais, seus subprodutos possuem imensa importância econômica, como na geração de energia, propiciando a redução de custos e beneficiando a sustentabilidade do setor.

Devido às extensas áreas cultivadas no Brasil e por ser um país com clima favorável, a cana é atacada por inúmeras pragas, das quais são responsáveis por grandes perdas nas lavouras. A cultura é a mais afetada com $54 \%$ de perdas pelo ataque de pragas, doenças e plantas daninhas. Desse total $20 \%$ são causados por pragas (DE JESUS, 2018).

Dentre elas, temos a Diatraea Saccharalis, conhecida popularmente como broca da cana de açúcar, é considerada a principal praga nas culturas de cana de açúcar, dado que possui uma ampla distribuição geográfica, está presente em todas as regiões produtoras de cana do Brasil. De acordo com (GALLO et al., 2002), a cada $1 \%$ de intensidade de infestação da praga, ocorrem prejuízos de $0,20 \%$ de álcool, $0,25 \%$ de açúcar e $0,77 \%$ da produtividade.

Existem diferentes métodos de controle que podem ser utilizados com o propósito de conter a pressão de pragas, entre eles estão o controle cultural, o biológico e o químico. 0 controle biológico é o mais usado no Brasil, ele se caracteriza em controlar pragas agrícolas com base no uso de seus inimigos naturais.

Um eficiente parasitoide é o Trichogramma Galloi que atua na fase do ovo da praga, tal fase é o fator principal de crescimento populacional da broca. Suas fêmeas localizam os ovos do hospedeiro. Colocam seus ovos, cessando o desenvolvimento da praga logo no inicio do seu ciclo, sintetizando rapidamente a população da praga, em consequência os prejuízos econômicos que seriam causados.

No controle biológico é significativo compreender a dinâmica da praga e seu inimigo natural. Uma ferramenta que auxilia nessa compreensão é a modelagem matemática, pois ela nos permite executar uma avaliação quantitativa e qualitativa visando o controle da praga. A modelagem matemática, de maneira resumida, é o método que envolve adquirir um modelo que consiga descrever matematicamente um fenômeno da nossa realidade, para fins de estudo e compreensão.

"A modelagem matemática consiste na arte de transformar problemas da realidade em problemas matemáticos e resolvê-los interpretando suas soluções na linguagem do mundo real" (BASSANEZI, 2002).

Assim, o objetivo deste artigo é analisar o modelo matemático que representa a interação da praga broca da cana de açúcar (Diatraea Saccharalis) com seu parasitoide Trichogramma Galloi, proposto por (RAFIKOV E LIMEIRA, 2010). Em sequência, analisar a estabilidade dos pontos de equilíbrio do sistema proposto. Por fim, determinar os parâmetros do sistema para exibir e analisar as simulações numéricas das interações realizadas.

Utilizamos o método Runge-Kutta de 4 a ordem no idioma $\mathrm{C}$, para resolver numericamente - sistema de equações (4), com amplitude $h=0,001$ e as condições iniciais dadas para o sistema.

\section{MODELO MATEMÁTICO}

O modelo matemático proposto neste artigo descreve a interação entre Diatraea Saccharalis, levando em consideração os seus dois principais estágios de desenvolvimento, os estágio de ovo e larval, e seu parasitoide Trichogramma Galloi.

Para construção do modelo consideremos $x_{1}, x_{2}$ e $x_{3}$ a densidade populacional de ovos da broca da cana, a densidade populacional de ovos parasitados e a densidade populacional de larvas, respectivamente.

A taxa de variação da densidade populacional de ovos $x_{1}$ é dada por:

$$
\begin{gathered}
\frac{d x_{1}}{d t}=\beta\left(1-\frac{x_{1}}{K}\right) x_{1}-m_{1} x_{1}-n_{1} x_{1} \\
-\alpha x_{1} x_{2}
\end{gathered}
$$

onde, $\beta$ é a taxa de reprodução de ovos, $K$ é a capacidade de suporte do meio que nunca se 
anula, $m_{1}$ é a taxa de mortalidade da população de ovos da broca, $n_{1}$ é o coeficiente que caracteriza a parte dos ovos da broca que se tornaram lagarta, no tempo $t$ e $\alpha$ é a taxa de parasitismo. Observamos que a taxa de crescimento dos ovos $x_{1}$ obedece a um crescimento logístico.

A taxa de variação da densidade populacional dos ovos $x_{2}$ é dada por:

$$
\frac{d x_{2}}{d t}=\alpha x_{1} x_{2}-m_{2} x_{2}-n_{2} x_{2},
$$

onde, $m_{2}$ é a taxa de mortalidade da população de ovos parasitados e $n_{2}$ é o coeficiente que caracteriza que parte dos ovos parasitados que se tornaram parasitoides, no tempo $t$.

A taxa de variação da densidade populacional de larvas $x_{3}$ é dada por:

$$
\frac{d x_{3}}{d t}=n_{1} m_{1}-m_{3} x_{3}-n_{3} x_{3},
$$

onde, $m_{3}$ é a taxa de mortalidade das larvas e $n_{3}$ é o coeficiente que caracteriza a parte de larvas que se tornaram pupas.

A partir das equações (1), (2) e (3) obtemos o seguinte sistema autônomo não linear de equações diferenciais ordinárias que modela a dinâmica da broca da cana e seu parasitoide:

$$
\left\{\begin{array}{c}
\frac{d x_{1}}{d t}=\beta\left(1-\frac{x_{1}}{K}\right) x_{1}-m_{1} x_{1}-n_{1} x_{1}-\alpha x_{1} x_{2} \\
\frac{d x_{2}}{d t}=\alpha x_{1} x_{2}-m_{2} x_{2}-n_{2} x_{2} \\
\frac{d x_{3}}{d t}=n_{1} x_{1}-m_{3} x_{3}-n_{3} x_{3} .
\end{array}\right.
$$

\section{PONTOS DE EQUILÍBRIO}

Observamos que o sistema (4) se trata de um sistema autônomo de equações não lineares e, em muitos casos, determinar explicitamente as soluções desse tipo de sistema não é uma tarefa muito fácil devido à dificuldade das equações diferenciais envolvidas. Portanto, uma alternativa é o estudo feito através de uma análise qualitativa do comportamento das soluções, ao invés de uma investigação quantitativa. $O$ estudo qualitativo de suas soluções é realizado através do seu sistema linearizado que é obtido aplicando o processo de linearização (DA SILVA, 2019).

Assim, para o estudo da estabilidade é preciso encontrar os pontos de equilíbrio do sistema (4), uma vez que, com base neles, é possível inferir qual será o comportamento da solução das equações diferenciais que modelam o sistema. Os pontos de equilíbrio são obtidos resolvendo o sistema algébrico:

$$
\left\{\begin{array}{c}
\beta\left(1-\frac{x_{1}^{*}}{K}\right) x_{1}^{*}-m_{1} x_{1}^{*}-n_{1} x_{1}^{*}-\alpha x_{1}^{*} x_{2}^{*}=0, \\
\alpha x_{1}^{*} x_{2}^{*}-m_{2} x_{2}^{*}-n_{2} x_{2}^{*}=0, \\
n_{1} x_{1}^{*}-m_{3} x_{3}^{*}-n_{3} x_{3}^{*}=0,
\end{array}\right.
$$

ou ainda, de forma mais simplificada:

$$
\left\{\begin{array}{c}
x_{1}^{*}\left[\left(\beta-\frac{\beta x_{1}^{*}}{K}\right)-m_{1}-n_{1}-\alpha x_{2}^{*}\right]=0, \\
x_{2}^{*}\left(\alpha x_{1}^{*}-m_{2}-n_{2}\right)=0, \\
n_{1} x_{1}^{*}-x_{3}^{*}\left(m_{3}+n_{3}\right)=0 .
\end{array} .\right.
$$

O primeiro ponto de equilíbrio é dado por:

$$
P_{1}=(0,0,0) \text {. }
$$

$\mathrm{Na}$ primeira equação do sistema (5) se supormos que $x_{1}^{*}$ é zero, estaríamos admitindo a não existência das pragas. Agora, tomando $x_{1}^{*} \neq 0$, temos:

$$
x_{1}^{*}=\frac{\left(-m_{1}-n_{1}-\alpha x_{2}+\beta\right) K}{\beta} .
$$

$\mathrm{Na}$ segunda equação do sistema (5), temos dois possíveis casos. $O$ primeiro é $x_{2}^{*}=0$, que ao substituir em (6):

$$
x_{1}^{*}=\frac{\left(-m_{1}-n_{1}+\beta\right) K}{\beta},
$$

e substituindo (7) na terceira equação do sistema (5), obtemos:

$$
x_{3}^{*}=\frac{n_{1}\left(-m_{1}-n_{1}+\beta\right) K}{\left(m_{3}+n_{3}\right) \beta} .
$$

Portanto, de (7), (8) e de $x_{2}^{*}=0$ temos o segundo ponto de equilíbrio dado por:

$$
\begin{gathered}
P_{2}= \\
\left(\frac{K}{\beta}\left(\beta-m_{1}-n_{1}\right), 0, \frac{n_{1} K\left(\beta-m_{1}-n_{1}\right)}{\beta\left(m_{3}+n_{3}\right)}\right) .
\end{gathered}
$$

Ainda na segunda equação o segundo caso possível é:

$$
x_{2}^{*}=\frac{\beta}{\alpha}-\frac{\beta\left(m_{2}+n_{2}\right)}{\alpha^{2} K}-\frac{m_{1}+n_{1}}{\alpha},
$$

substituindo (9) em (6), temos que:

$$
x_{1}^{*}=\frac{m_{2}+n_{2}}{\alpha},
$$

e assim, substituindo (10) na terceira equação de (6) teremos:

$$
x_{3}^{*}=\frac{n_{1}\left(m_{2}+n_{2}\right)}{\left(m_{3}+n_{3}\right) \alpha} .
$$

Portanto, o terceiro ponto de equilíbrio é dado por:

$$
\begin{aligned}
P_{3}=( & \frac{m_{2}+n_{2}}{\alpha}, \frac{\beta}{\alpha}-\frac{\beta\left(m_{2}+n_{2}\right)}{\alpha^{2} K} \\
& \left.-\frac{m_{1}+n_{1}}{\alpha}, \frac{n_{1}\left(m_{2}+n_{2}\right)}{\left(m_{3}+n_{3}\right) \alpha}\right) .
\end{aligned}
$$

Por se tratar de um modelo de praga é preciso garantir que a população não seja 
negativa, pois não tem sentido biológico. Em vista disso, no ponto $P_{2}$ a condição necessária é:

$$
\begin{gathered}
\beta-m_{1}-n_{1}>0, \\
\beta>m_{1}+n_{1},
\end{gathered}
$$

e no ponto $P_{3}$ a condição necessária é:

$$
\begin{gathered}
\frac{\beta}{\alpha}-\frac{\beta\left(m_{2}+n_{2}\right)}{\alpha^{2} K}-\frac{m_{1}-n_{1}}{\alpha}>0, \\
\beta>\frac{\left(m_{1}+n_{1}\right) \alpha K}{\alpha K-\left(m_{2}+n_{2}\right)} .
\end{gathered}
$$

\section{ANÁLISE DE ESTABILIDADE}

Para sistemas não lineares o processo algébrico para inferir a estabilidade dos pontos de equilíbrio é fundamentado no estudo dos autovalores da matriz jacobiana do sistema. Seja $X^{*}=\left(x_{1}^{*}, x_{2}^{*}, x_{3}^{*}\right)$ um ponto de equilíbrio de (4). 0 Teorema de Hartman Grobman enuncia que a estabilidade de $X^{*}$ e o retrato de fase do sistema (4), localmente em torno de $X^{*}$, podem ser conhecidos através da linearização do sistema (4) em torno de $X^{*}$.

Teorema de Hartman Grobman. Dado o sistema não linear,

$$
\left\{\begin{array}{l}
\frac{d x_{1}}{d t}=F\left(x_{1}, x_{2}, x_{3}\right), \\
\frac{d x_{2}}{d t}=G\left(x_{1}, x_{2}, x_{3}\right), \\
\frac{d x_{3}}{d t}=H\left(x_{1}, x_{2}, x_{3}\right),
\end{array}\right.
$$

se as condições 1 e 2 a seguir são satisfeitas, então o retrato de fase do sistema não linear (12), localmente em torno dos pontos de equilíbrio, é equivalente ao retrato de fase do sistema linearizado nos pontos de equilíbrio, dado por:

$$
\left\{\begin{array}{l}
\frac{d x_{1}}{d t}=a x_{1}+b x_{2}+c x_{3}, \\
\frac{d x_{2}}{d t}=d x_{1}+e x_{2}+f x_{3}, \\
\frac{d x_{3}}{d t}=g x_{1}+h x_{2}+i x_{3},
\end{array}\right.
$$

onde,

$$
\begin{aligned}
& a=F_{x_{1}}\left(x_{1}^{*}, x_{2}^{*}, x_{3}^{*}\right), \\
& b=F_{x_{2}}\left(x_{1}^{*}, x_{2}^{*}, x_{3}^{*}\right), \\
& c=F_{x_{3}}\left(x_{1}^{*}, x_{2}^{*}, x_{3}^{*}\right), \\
& d=G_{x_{1}}\left(x_{1}^{*}, x_{2}^{*}, x_{3}^{*}\right), \\
& e=G_{x_{2}}\left(x_{1}^{*}, x_{2}^{*}, x_{3}^{*}\right), \\
& f=G_{x_{3}}\left(x_{1}^{*}, x_{2}^{*}, x_{3}^{*}\right), \\
& g=H_{x_{1}}\left(x_{1}^{*}, x_{2}^{*}, x_{3}^{*}\right), \\
& h=H_{x_{2}}\left(x_{1}^{*}, x_{2}^{*}, x_{3}^{*}\right), \\
& i=H_{x_{3}}\left(x_{1}^{*}, x_{2}^{*}, x_{3}^{*}\right),
\end{aligned}
$$

Em notação matricial:

$$
X^{\prime}=A X,
$$

onde A é a matriz jacobiana do sistema calculado no ponto $\left(x_{1}^{*}, x_{2}^{*}, x_{3}^{*}\right)$ :

$$
A=J\left(x_{1}^{*}, x_{2}^{*}, x_{3}^{*}\right)=\left(\begin{array}{lll}
\frac{\partial F}{\partial x_{1}} & \frac{\partial F}{\partial x_{2}} & \frac{\partial F}{\partial x_{3}} \\
\frac{\delta G}{\partial x_{1}} & \frac{\delta G}{\partial x_{2}} & \frac{\delta G}{\partial x_{3}} \\
\frac{\delta H}{\partial x_{1}} & \frac{\delta H}{\partial x_{2}} & \frac{\delta H}{\partial x_{3}}
\end{array}\right) .
$$

As condições são:

1) $O$ determinante de $A=J\left(x_{1}^{*}, x_{2}^{*}, x_{3}^{*}\right) \neq 0$,

2) Cada autovalor de $A$ deve possui parte real não nula.

Portanto, para construir a matriz jacobiana do sistema (4), consideramos:

$$
\begin{gathered}
F\left(x_{1}, x_{2}, x_{3}\right)=\beta\left(1-\frac{x_{1}}{K}\right) x_{1}-m_{1} x_{1} \\
-n_{1} x_{1}-\alpha x_{1} x_{2}, \\
G\left(x_{1}, x_{2}, x_{3}\right)=\alpha x_{1} x_{2}-m_{2} x_{2}-n_{2} x_{2}, \\
H\left(x_{1}, x_{2}, x_{3}\right)=n_{1} x_{1}-m_{3} x_{3}-n_{3} x_{3} .
\end{gathered}
$$

Agora, é preciso calcular as derivadas parciais de cada função acima no ponto de equilíbrio. Em (13):

$$
\begin{gathered}
F_{x_{1}}\left(x_{1}^{*}, x_{2}^{*}, x_{3}^{*}\right)=\beta-\frac{2 \beta x_{1}^{*}}{K}-m_{1}-\alpha x_{2}^{*}, \\
F_{x_{2}}\left(x_{1}^{*}, x_{2}^{*}, x_{3}^{*}\right)=-\alpha x_{1}^{*}, \\
F_{x_{3}}\left(x_{1}^{*}, x_{2}^{*}, x_{3}^{*}\right)=0 .
\end{gathered}
$$

$\operatorname{Em}(14)$,

$$
\begin{gathered}
G_{x_{1}}\left(x_{1}^{*}, x_{2}^{*}, x_{3}^{*}\right)=\alpha x_{2}^{*}, \\
G_{x_{2}}\left(x_{1}^{*}, x_{2}^{*}, x_{3}^{*}\right)=\alpha x_{1}^{*}-m_{2}-n_{2}, \\
G_{x_{3}}\left(x_{1}^{*}, x_{2}^{*}, x_{3}^{*}\right)=0 .
\end{gathered}
$$

Em (15),

$$
\begin{gathered}
H_{x_{1}}\left(x_{1}^{*}, x_{2}^{*}, x_{3}^{*}\right)=n_{1}, \\
H_{x_{2}}\left(x_{1}^{*}, x_{2}^{*}, x_{3}^{*}\right)=0, \\
H_{x_{3}}\left(x_{1}^{*}, x_{2}^{*}, x_{3}^{*}\right)=-m_{3}-n_{3} .
\end{gathered}
$$

Construindo a matriz jacobiana do sistema:

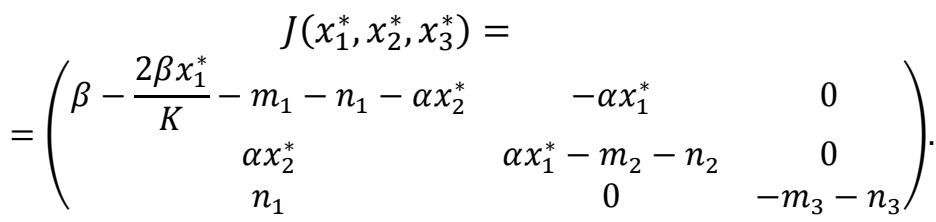

Portanto, para o cálculo da estabilidade dos pontos de equilíbrio, basta estudar os autovalores do sistema linearizado:

$$
\left\{\begin{array}{c}
\frac{d x_{1}}{d t}=\left[\beta\left(1-\frac{2 x_{1}^{*}}{K}\right)-m_{1}-n_{1}-\alpha x_{2}^{*}\right] x_{1}+\left(-\alpha x_{1}^{*}\right) x_{2}+0 x_{3}, \\
\frac{d x_{2}}{d t}=\left(\alpha x_{2}^{*}\right) x_{1}+\left(\alpha x_{1}^{*}-m_{2}-n_{2}\right) x_{2}+0 x_{3}, \\
\frac{d x_{3}}{d t}=n_{1} x_{1}+0 x_{2}+\left(-m_{3}-n_{3}\right) x_{3} .
\end{array}\right.
$$


Para o cálculo dos autovalores da matriz (16) façamos $\operatorname{det}(J-\lambda I)=0$ :

$\left|\begin{array}{ccc}\beta-\frac{2 \beta x_{1}^{*}}{K}-m_{1}-n_{1}-\alpha x_{2}^{*}-\lambda & -\alpha x_{1}^{*} & 0 \\ \alpha x_{2}^{*} & \alpha x_{1}^{*}-m_{2}-n_{2}-\lambda & 0 \\ n_{1} & 0 & -m_{3}-n_{3}-\lambda\end{array}\right|$

onde, obtemos:

$$
\begin{gathered}
\left(-m_{3}-n_{3}-\lambda\right)\left\{\left[\beta\left(1-\frac{2 x_{1}^{*}}{K}\right)-m_{1}-n_{1}\right.\right. \\
\left.-\alpha x_{2}^{*}-\lambda\right]\left[\alpha x_{1}^{*}-m_{2}-n_{2}\right. \\
\left.-\lambda]+\alpha^{2} x_{1}^{*} x_{2}^{*}\right\}=0 .
\end{gathered}
$$

Assim:

$$
-m_{3}-n_{3}-\lambda=0
$$

ou,

$$
\begin{aligned}
& {\left[\beta\left(1-\frac{2 x_{1}^{*}}{K}\right)-m_{1}-n_{1}-\alpha x_{2}^{*}-\lambda\right]} \\
& {\left[\alpha x_{1}^{*}-m_{2}-n_{2}-\lambda\right]+\alpha^{2} x_{1}^{*} x_{2}^{*}=0 .}
\end{aligned}
$$

(16) é:

Portanto, um dos autovalores da matriz

$$
\lambda_{1}=-m_{3}-n_{3} \text {. }
$$

Na equação (17),

$$
\begin{gathered}
{\left[\beta\left(1-\frac{2 x_{1}^{*}}{K}\right)-m_{1}-n_{1}-\alpha x_{2}^{*}\right]} \\
\left(\alpha x_{1}^{*}-m_{2}-n_{2}\right)- \\
{\left[\beta\left(1-\frac{2 x_{1}^{*}}{K}\right)-m_{1}-n_{1}-\alpha x_{2}^{*}\right] \lambda-} \\
\lambda\left(\alpha x_{1}^{*}-m_{2}-n_{2}\right)+\lambda^{2}+\alpha^{2} x_{1}^{*} x_{2}^{*}=0,
\end{gathered}
$$

ou, ainda,

$$
\begin{aligned}
\lambda^{2}+ & {\left[-\beta\left(1-\frac{2 x_{1}^{*}}{K}\right)+m_{1}+n_{1}+\alpha x_{2}^{*}-\alpha x_{1}^{*}\right.} \\
& \left.+m_{2}+n_{2}\right] \lambda+ \\
& {\left[\beta\left(1-\frac{2 x_{1}^{*}}{K}\right)-m_{1}-n_{1}-\alpha x_{2}^{*}\right] } \\
& \left(\alpha x_{1}^{*}-m_{2}-n_{2}\right)+\alpha^{2} x_{1}^{*} x_{2}^{*}=0 . \\
& \text { Logo, os outros autovalores da matriz }
\end{aligned}
$$

(16) podem ser obtidos através da equação:

$$
a_{0} \lambda^{2}+a_{1} \lambda+a_{2}=0 \text {, }
$$

onde,

$$
\begin{gathered}
a_{0}=1, \\
a_{1}=\left[-\beta\left(1-\frac{2 x_{1}^{*}}{K}\right)+m_{1}+n_{1}+\alpha x_{2}^{*}-\alpha x_{1}^{*}\right. \\
\left.+m_{2}+n_{2}\right], \\
a_{2}=\left[\beta\left(1-\frac{2 x_{1}^{*}}{K}\right)-m_{1}-n_{1}-\alpha x_{2}^{*}\right] \\
\left(\alpha x_{1}^{*}-m_{2}-n_{2}\right)+\alpha^{2} x_{1}^{*} x_{2}^{*} .
\end{gathered}
$$

Agora, analisaremos cada ponto de equilíbrio por vez. Para a análise de estabilidade do ponto de equilíbrio:

$$
P_{1}=(0,0,0),
$$

basta substituir os valores do ponto de equilíbrio $P_{1}$ na matriz jacobiana (16), e assim obtemos:

$$
J_{1}=\left(\begin{array}{ccc}
\beta-m_{1}-n_{1} & 0 & 0 \\
0 & -m_{2}-n_{2} & 0 \\
n_{1} & 0 & -m_{3}-n_{3}
\end{array}\right) \text {. }
$$

Determinemos os autovalores de $J_{1}$ encontrando os zeros do polinômio característico, isto é, $\operatorname{det}\left(\mathrm{J}_{1}-\lambda \mathrm{I}\right)=0$. Então,

$$
\left|\begin{array}{ccc}
\beta-m_{1}-n_{1}-\lambda & 0 & 0 \\
0 & -m_{2}-n_{2}-\lambda & 0 \\
n_{1} & 0 & -m_{3}-n_{3}-\lambda
\end{array}\right|
$$

por se tratar de uma matriz triangular inferior, o determinante desta é calculado através do produto dos elementos da diagonal, ou seja:

$$
\begin{gathered}
\left(\beta-m_{1}-n_{1}-\lambda\right)\left(-m_{2}-n_{2}-\lambda\right) \\
\left(-m_{3}-n_{3}-\lambda\right)=0 .
\end{gathered}
$$

Assim:

$$
\begin{gathered}
-m_{3}-n_{3}-\lambda=0, \\
\beta-m_{1}-n_{1}-\lambda=0, \\
-m_{2}-n_{2}-\lambda=0 .
\end{gathered}
$$

onde obtemos os seguintes autovalores:

$$
\begin{gathered}
\lambda_{1}=-m_{3}-n_{3}, \\
\lambda_{2}=\beta-m_{1}-n_{1}, \\
\lambda_{3}=-m_{2}-n_{2} .
\end{gathered}
$$

Para o ponto $P_{1}$ ser estável os autovalores devem ser negativos (MATTIUZZO, 2016), sendo assim devemos ter as seguintes condições:

$$
\begin{gathered}
\beta-m_{1}-n_{1}<0, \\
\beta<m_{1}+n_{1} .
\end{gathered}
$$

Para análise de estabilidade do ponto de equilíbrio:

$$
\begin{gathered}
P_{2}= \\
\left(\frac{K}{\beta}\left(\beta-m_{1}-n_{1}\right), 0, \frac{n_{1} K\left(\beta-m_{1}-n_{1}\right)}{\beta\left(m_{3}+n_{3}\right)}\right),
\end{gathered}
$$

basta substituir os valores do ponto de equilíbrio $P_{2}$ na matriz jacobiana (16), e assim obtemos:

$$
\left.\begin{array}{ccc}
J_{2}= & \\
\beta\left(1-\frac{2 x_{1}^{*}}{K}\right)-m_{1}-n_{1} & -\alpha x_{1}^{*} & 0 \\
0 & \alpha x_{1}^{*}-m_{2}-n_{2} & 0 \\
n_{1} & 0 & -m_{3}-n_{3}
\end{array}\right) .
$$

Determinemos os autovalores de $J_{2}$ encontrando os zeros do polinômio característico, $\operatorname{det}\left(J_{2}-\lambda I\right)=0$,

$$
\begin{aligned}
& \left|\begin{array}{ccc}
\beta\left(1-\frac{2 x_{1}^{*}}{K}\right)-m_{1}-n_{1}-\lambda & -\alpha x_{1}^{*} & 0 \\
0 & \alpha x_{1}^{*}-m_{2}-n_{2}-\lambda & 0 \\
n_{1} & 0 & -m_{3}-n_{3}-\lambda
\end{array}\right| \\
& =0 \text {, }
\end{aligned}
$$

onde, obtemos:

$$
\begin{gathered}
\left\{\beta\left(1-\frac{2 x_{1}^{*}}{K}\right)-m_{1}-n_{1}-\lambda\right\}\left(\alpha x_{1}^{*}-m_{2}-n_{2}\right. \\
-\lambda)\left(-m_{3}-n_{3}-\lambda\right)=0 . \\
\text { Assim: }
\end{gathered}
$$

$$
-m_{3}-n_{3}-\lambda=0
$$




$$
\begin{gathered}
\beta\left(1-\frac{2 x_{1}^{*}}{K}\right)-m_{1}-n_{1}-\lambda=0, \\
\alpha x_{1}^{*}-m_{2}-n_{2}-\lambda=0
\end{gathered}
$$

substituindo as coordenadas do ponto de equilíbrio $P_{2}$ nas equações (20), obtemos:

$$
\begin{gathered}
\beta\left(1-\frac{2}{\beta}\left(\beta-m_{1}-n_{1}\right)-m_{1}-n_{1}-\lambda=0,\right. \\
-\beta+m_{1}+n_{1}-\lambda=0,
\end{gathered}
$$

e em (21), temos:

$$
\begin{gathered}
\alpha\left\{\frac{K}{\beta}\left(\beta-m_{1}-n_{1}\right)\right\}-m_{2}-n_{2}-\lambda=0, \\
\frac{\alpha K}{\beta}\left(\beta-m_{1}-n_{1}\right)-m_{2}-n_{2}-\lambda=0 .
\end{gathered}
$$

Assim, obtemos os seguintes autovalores:

$$
\begin{gathered}
\lambda_{1}=-m_{3}-n_{3}, \\
\lambda_{2}=-\beta+m_{1}+n_{1}, \\
\lambda_{3}=\frac{\alpha K}{\beta}\left(\beta-m_{1}-n_{1}\right)-m_{2}-n_{2} .
\end{gathered}
$$

Para o ponto $P_{2}$ ser estável é preciso que seja satisfeita as condições:

$$
\beta>m_{1}+n_{1}
$$

e,

$$
\beta<\frac{\alpha K\left(m_{1}+n_{1}\right)}{\alpha K-\left(m_{2}+n_{2}\right)} .
$$

Para análise de estabilidade do ponto de equilíbrio:

$$
\begin{aligned}
P_{3}=( & \frac{m_{2}+n_{2}}{\alpha}, \frac{\beta}{\alpha}-\frac{\beta\left(m_{2}+n_{2}\right)}{\alpha^{2} K} \\
& \left.-\frac{m_{1}+n_{1}}{\alpha}, \frac{n_{1}\left(m_{2}+n_{2}\right)}{\left(m_{3}+n_{3}\right) \alpha}\right),
\end{aligned}
$$

basta substituir os valores do ponto de equilíbrio $P_{3}$ na matriz jacobiana (16), e assim obtemos:

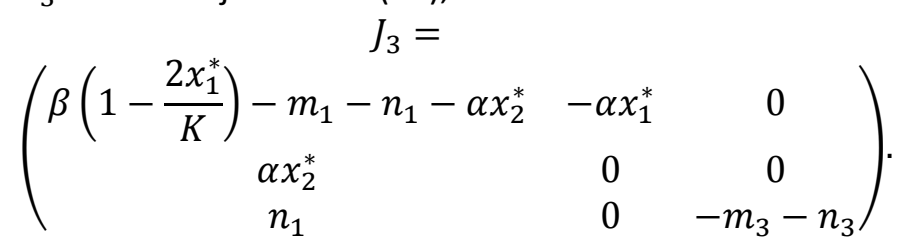

Determinemos os autovalores de $J_{3}$ encontrando os zeros do polinômio característico, $\operatorname{det}\left(J_{3}-\lambda I\right)=0$, logo:

$$
\begin{aligned}
& \left|\begin{array}{ccc}
\beta\left(1-\frac{2 x_{1}^{*}}{K}\right)-m_{1}-n_{1}-\alpha x_{2}^{*}-\lambda & -\alpha x_{1}^{*} & 0 \\
\alpha x_{2}^{*} & -\lambda & 0 \\
n_{1} & 0 & -m_{3}-n_{3}-\lambda
\end{array}\right| \\
& =0 \text {, }
\end{aligned}
$$

onde, obtemos:

$$
\begin{gathered}
\left\{\beta\left(1-\frac{2 x_{1}^{*}}{K}\right)-m_{1}-n_{1}-\alpha x_{2}^{*}-\lambda\right\} \\
(-\lambda)\left(-m_{3}-n_{3}-\lambda\right)- \\
\left\{\left(-\alpha x_{1}^{*}\right)\left(\alpha x_{2}^{*}\right)\left(-m_{3}-n_{3}-\lambda\right)\right\}=0 \\
\text { Pelas equações (18) e (19) sabemos que }
\end{gathered}
$$
um dos autovalores é:

$$
\lambda_{1}=-m_{3}-n_{3},
$$

e os outros podem ser obtidos a partir da equação:

$$
a_{0} \lambda^{2}+a_{1} \lambda+a_{2}=0
$$

onde os coeficientes, substituindo as coordenadas do ponto de equilíbrio $P_{3}$, são dados por:

$$
\begin{gathered}
a_{0}=1, \\
a_{1}=-\beta\left(1-\frac{2\left(m_{2}+n_{2}\right)}{\alpha K}\right)+n_{1}+ \\
\left\{\beta-\frac{\beta\left(m_{2}-n_{2}\right)}{\alpha K}-m_{1}-n_{1}\right\}- \\
n_{2}-m_{2}+m_{2}+n_{2}=\frac{\beta\left(m_{2}+n_{2}\right)}{\alpha K}, \\
a_{2}= \\
\alpha\left\{\beta-\frac{\beta\left(m_{2}+n_{2}\right)}{\alpha K}-\left(m_{1}+n_{1}\right)\right\} \\
\left\{\beta-\beta\left(\frac{m_{2}+n_{2}}{\alpha K}\right)-\left(m_{1}+n_{1}\right)\right\} \\
\left(m_{2}+n_{2}\right) .
\end{gathered}
$$

Como a equação é um tanto complexa, uma forma de analisar a estabilidade dos autovalores é utilizar o critério de estabilidade de Routh; com ele não é necessário calcular os autovalores. Tal critério se baseia em analisar os coeficientes da equação característica e estabelecer condições para que possamos concluir se o ponto é estável ou não (DE CAMPOS, [s.d]).

O critério de Routh mostra que a condição necessária e suficiente para a estabilidade é que todos os elementos na primeira coluna da tabela de Routh sejam positivos. A tabela de Routh possui $n+1$ linha, onde $n$ é o grau do polinômio. As duas primeiras linhas são preenchidas com os coeficientes do polinômio da esquerda para a direita, alternando entre as linhas. Para o caso do polinômio (22), a tabela 1 ilustra o procedimento.

Tabela 1. Tabela de Routh.

\begin{tabular}{c|c|c}
\hline & & $\left\{\beta-\beta\left(\frac{m_{2}+n_{2}}{\alpha K}\right)\right.$ \\
$\lambda^{2}$ & 1 & $\begin{array}{l}\left.\left\{m_{1}+n_{1}\right)\right\}\left(m_{2}\right. \\
\left.+n_{2}\right)\end{array}$ \\
\hline$\lambda^{1}$ & $\frac{\beta\left(m_{2}+n_{2}\right)}{\alpha K}$ & \\
\hline$\lambda^{0}$ & $d_{1}$ & \\
\hline
\end{tabular}

Fonte: $\mathrm{O}$ autor.

Em seguida a tabela é preenchida, determinando o valor de $d_{1}$, a partir do seguinte cálculo:

$$
d_{1}=
$$




$$
\begin{gathered}
\frac{\left\{\left[\beta-\beta\left(\frac{m_{2}+n_{2}}{\alpha K}\right)-\left(m_{1}+n_{1}\right)\right]\left(m_{2}+n_{2}\right)\right\} \times\left\{\frac{\beta\left(m_{2}+n_{2}\right)}{\alpha K}\right\}-1 \times 0}{\frac{\beta\left(m_{2}+n_{2}\right)}{\alpha K}} \\
d_{1}=\left\{\beta-\beta\left(\frac{m_{2}+n_{2}}{\alpha K}\right)-\left(m_{1}+n_{1}\right)\right\} \\
\left(m_{2}+n_{2}\right)
\end{gathered}
$$

Para que tenhamos a estabilidade, temos que garantir que os elementos da primeira coluna sejam positivos, ou seja:

$$
a_{1}>0 \text { e } a_{2}>0 \text {. }
$$

Pela equação (23), já temos que $a_{1}>0$, pois:

$$
\frac{\beta\left(m_{2}+n_{2}\right)}{\alpha K}>0 .
$$

$\mathrm{Na}$ equação (24), para $a_{2}>0$ é necessário que:

$$
\begin{gathered}
\beta-\beta\left(\frac{m_{2}+n_{2}}{\alpha K}\right)-\left(m_{1}+n_{1}\right)>0, \\
\beta\left(1-\frac{m_{2}+n_{2}}{\alpha K}\right)>\left(m_{1}+n_{1}\right), \\
\beta>\frac{\left(m_{1}+n_{1}\right)}{\left(1-\frac{m_{2}+n_{2}}{\alpha K}\right)}, \\
\beta>\frac{\left(m_{1}+n_{1}\right) \alpha K}{\alpha K-\left(m_{2}+n_{2}\right)} .
\end{gathered}
$$

Finalmente, encerramos a análise de estabilidade dos pontos de equilíbrios. A seguir, antes de executar as simulações numéricas é necessário determinar os parâmetros que serão utilizados.

\section{DETERMINAÇÃO DOS PARÂMETROS}

De acordo com (POLETTI et al., 2019), a duração de vida dos ovos normais da broca é de 4 a 9 dias e a duração do estágio larval é de 40 a 60 dias. Segundo (PEREIRA-BARROS et al., 2005), a emergência dos ovos parasitados ocorre em até 10 dias. O tempo médio de vida de cada fase, considerando a média entre esses dados, está retratado na tabela 2 .

Tabela 2. Tempo médio de vida.

\begin{tabular}{c|c|c|c}
\hline Fase & $\begin{array}{c}\text { Ovo } \\
\text { normal }\end{array}$ & $\begin{array}{c}\text { Ovo } \\
\text { parasitado }\end{array}$ & Lagarta \\
\hline $\begin{array}{c}\text { Duração } \\
\text { (dias) }\end{array}$ & 8 & 8 & 50 \\
\hline
\end{tabular}

Fonte: $\mathrm{O}$ autor.

Consideramos $t_{m_{1}}, t_{m_{2}}$ e $t_{m_{3}} \circ$ tempo médio de vida dos ovos normais, ovos parasitados e lagartas, respectivamente. Para determinar os parâmetros $n_{1}, n_{2}, n_{3}$ façamos:

$$
n_{1}=\frac{1}{t_{m_{1}}}=\frac{1}{8}=0,125
$$

$$
\begin{aligned}
& n_{2}=\frac{1}{t_{m_{2}}}=\frac{1}{8}=0,125, \\
& n_{3}=\frac{1}{t_{m_{3}}}=\frac{1}{50}=0,02 .
\end{aligned}
$$

De acordo com (AMBROSANO, 1994), a mortalidade dos ovos normais da broca, das lagartas e dos ovos parasitados variam, respectivamente, em torno de: 70\%, 90\% e 42,3\%. Conforme (SHEIL et al., 1995), os parâmetros $m_{1}, m_{2}$ e $m_{3}$ são calculados por:

$$
\begin{gathered}
m_{1}=-\frac{\ln (0,7)}{t_{m_{1}}}=\frac{0,35667}{8}=0,04458375, \\
m_{2}=-\frac{\ln (0,423)}{t_{m_{2}}}=\frac{0,86038}{8}=0,1075475, \\
m_{3}=-\frac{\ln (0,9)}{t_{m_{3}}}=\frac{0,10536}{50}=0,0021072 .
\end{gathered}
$$

São poucos os trabalhos que estudam a dinâmica de interação entre a broca e seus inimigos naturais e dentre eles ainda faltam algumas reparações dos parâmetros utilizados para que tenhamos mais adequação com a realidade, por exemplo, subestimar a capacidade do meio ambiente. Portanto, a capacidade do meio considerada neste artigo está de acordo com (RAFIKOV E LIMEIRA, 2012):

$$
K=25000 \text {. }
$$

O nível de parasitismo do Trichogramma Galloi varia de acordo com a densidade da população da broca e ainda está associado ao estado fenológico da cultura (AMBROSANO, 1994). Desta forma, neste artigo a taxa de parasitismo considerada está conforme (RAFIKOV, 2010):

$$
\alpha=0,0002696 \text {. }
$$

Portanto, os valores dos parâmetros utilizados para a realização das simulações numéricas estão apresentados na tabela 3 a seguir:

Tabela 3. Parâmetros.

\begin{tabular}{c|c}
\hline Representação & Valor \\
\hline$m_{1}$ & 0,04458375 \\
\hline$m_{2}$ & 0,1075475 \\
\hline$m_{3}$ & 0,0021072 \\
\hline$n_{1}$ & 0,125 \\
\hline$n_{2}$ & 0,125 \\
\hline$n_{3}$ & 0,02 \\
\hline$\alpha$ & 0,0002696 \\
\hline $\mathrm{K}$ & 25000
\end{tabular}

Fonte: $\mathrm{O}$ autor.

As condições iniciais utilizadas para realizar as simulações numéricas foram 
$x_{1}=1000, x_{2}=1000$ e $x_{3}=1000$. Utilizamos o método Runge-Kutta de 4 o ordem no idioma $C$ para obtermos a solução discreta do sistema (4) e podermos comparar as soluções estabilizadas.

Obtidos os valores dos coeficientes, primeiramente é necessário calcular o determinante da matriz jacobiana em cada ponto de equilíbrio para que possamos validar o teorema de Hartman Grobman. $O$ determinante da matriz $J_{1}$ no ponto de equilíbrio $P_{1}$ é dado por:

$$
\begin{aligned}
\operatorname{det}\left(J_{1}\right)= & \left(\beta-m_{1}-n_{1}\right)\left(-m_{2}-n_{2}\right) \\
& \left(-m_{3}-n_{3}\right),
\end{aligned}
$$

substituindo os valores dos parâmetros já obtidos, temos:

$$
\operatorname{det}\left(J_{1}\right)=-4,926981045 \times 10^{-5} \neq 0 .
$$

No ponto de equilíbrio $P_{2}$ o determinante da matriz $J_{2}$ é dado por:

$$
\begin{gathered}
\operatorname{det}\left(J_{2}\right)=\left\{\beta\left(1-\frac{2 x_{1}^{*}}{K}\right)-m_{1}-n_{1}\right\}\left(\alpha x_{1}^{*}-m_{2}\right. \\
\left.-n_{2}\right) \\
\left(-m_{3}-n_{3}\right),
\end{gathered}
$$

substituindo os valores dos parâmetros já obtidos, temos:

$$
\operatorname{det}\left(J_{2}\right)=1,104490406 \times 10^{-5} \neq 0 .
$$

No ponto de equilíbrio $P_{3}$ o determinante da matriz $J_{3}$ é dado por:

$$
\operatorname{det}\left(J_{3}\right)=-\left\{\left(-\alpha x_{1}^{*}\right)\left(\alpha x_{2}^{*}\right)\left(-m_{3}-n_{3}\right)\right\}
$$

substituindo os valores dos parâmetros já obtidos, temos:

$$
\operatorname{det}\left(J_{3}\right)=-0,00160684326 \neq 0 .
$$

Portanto, como o determinante de $J_{1}, J_{2}, J_{3} \neq 0$, a segunda condição do teorema de Hartman Grobman está satisfeita. Os parâmetros foram determinados de forma que os autovalores fossem estritamente menores que zero, satisfazendo assim a primeira condição do teorema. Dessa forma, tornamos válido o teorema de Hartman Grobman para o sistema não linear (4).

\section{SIMULAÇÕES NÚMERICAS}

Para a análise de estabilidade do ponto de equilíbrio $P_{1}=(0,0,0)$ o coeficiente $\beta$ deve satisfazer a condição:

ou seja,

$$
\beta<m_{1}+n_{1}
$$

$$
\beta<0,16958375 \text {. }
$$

Desta forma, tomando $\beta=0,16$ e $\alpha=0,0002696$ a figura 1 exibe o comportamento das soluções.
Figura 1. Evolução das populações.

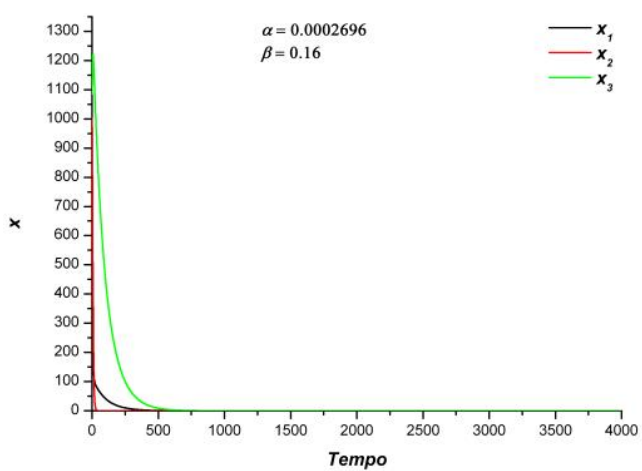

Fonte: $\mathrm{O}$ autor.

Nestas condições o ponto $P_{1}$ é estável. Para $t$ suficientemente grande as populações tendem a se extinguir conforme ilustra figura 1.

Para análise de estabilidade do ponto de equilíbrio

$$
P_{2}=(61,2132 ; 0 ; 346,1159),
$$

o coeficiente $\beta$ deve satisfazer a condição:

ou seja,

$$
m_{1}+n_{1}<\beta<\frac{\alpha K\left(m_{1}+n_{1}\right)}{\alpha K-\left(m_{2}+n_{2}\right)}
$$

$0,16958375<\beta<0,17564392133$.

Desta forma, tomando $\beta=0,17$ e $\alpha=0,0002696$ a figura 2 exibe o comportamento das soluções.

Figura 2. Evolução das populações.

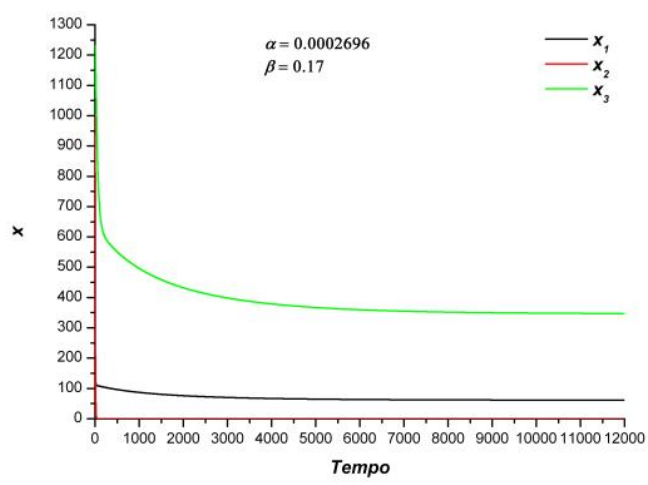

Fonte: $\mathrm{O}$ autor.

Nestas condições o ponto $P_{2}$ é estável. Por outro lado, a figura 3 ilustra também a estabilidade do ponto $P_{2}$, mas com o gráfico de uma forma ampliada, sem alterar os dados, ficando mais fácil verificar que para $t$ suficientemente grande a população de ovos parasitados tende a se extinguir, ao passo que a densidade dos ovos normais e lagartas tendem a se estabilizar. 
Figura 3. Evolução das populações.

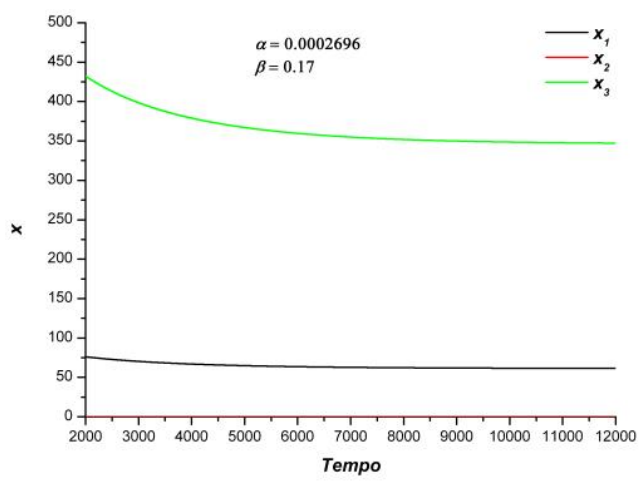

Fonte: $\mathrm{O}$ autor.

Para a análise de estabilidade do ponto de equilíbrio

$P_{3}=(862,5649 ; 15,600 ; 4877,1788)$, o coeficiente $\beta$ deve satisfazer a condição:

ou seja,

$$
\beta>\frac{\alpha K\left(m_{1}+n_{1}\right)}{\alpha K-\left(m_{2}+n_{2}\right)}
$$

$$
\beta>0,17564392133 .
$$

Desta forma, tomando $\beta=0,18$ e $\alpha=0,0002696$ a figura 3 exibe 0 comportamento das soluções.

Figura 4. Evolução das populações.

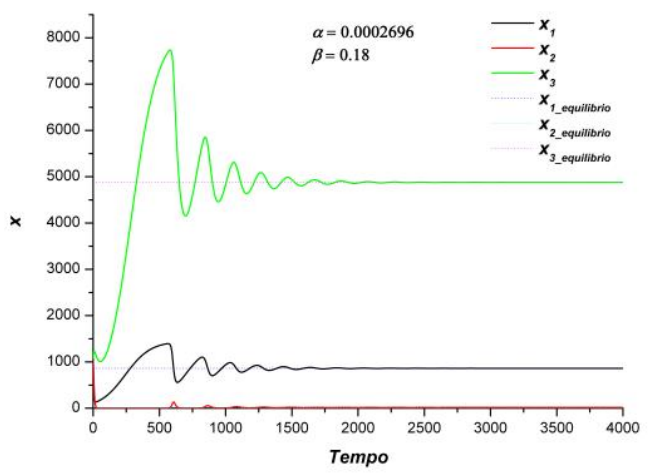

Fonte: $\mathrm{O}$ autor.

Nestas condições o ponto $P_{3}$ é estável. Por outro lado, a figura 5 ilustra também a estabilidade do ponto $P_{3}$, mas com o gráfico de uma forma ampliada, sem alterar os dados, facilitando averiguar que para $t$ suficientemente grande as populações de ovos normais, ovos parasitados e lagartas tendem a se estabilizar e coexistir no mesmo ambiente.
Figura 5. Evolução das populações.

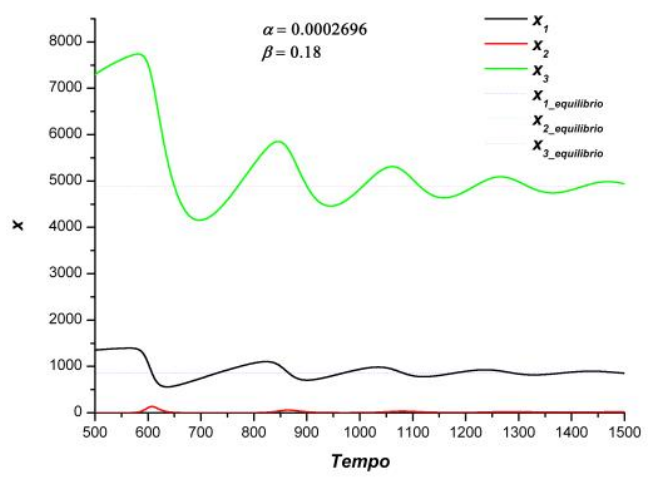

Fonte: $\mathrm{O}$ autor.

\section{CONSIDERAÇÕES FINAIS}

Estudar e compreender a dinâmica de interação entre a população da Diatraea Saccharalis e o seu parasitoide Trichogramma Galloi por meio da modelagem matemática e simulações numéricas é de suma importância, visto que podem contribuir consideravelmente no sucesso do controle da praga. Por exemplo, na figura 4 observamos que a população da broca da cana apresenta valores maiores que o limite de 2500 pragas por hectare, valores acima desse nível provocam prejuízos econômicos à cultura, portanto nesse cenário seria necessário aplicar o controle biológico.

As condições iniciais utilizadas neste artigo não se baseiam em situações reais de campo. Os estados de equilíbrio e os eventos são influenciados pelos coeficientes do modelo e também as variáveis consideradas na simulação numérica alteram a dinâmica de interação, o que revela a importância da adaptação dessas variáveis para a situação particular do produtor. Além disso, observamos que um parâmetro importante que interfere na estabilidade do ponto de equilíbrio é a taxa de reprodução $\beta$.

Neste modelo, encontramos os pontos de equilíbrio e condições para a estabilidade de cada um deles, e podemos observar que as simulações numéricas mostram uma boa aproximação para a estabilidade.

\section{AGRADECIMENTOS}

Agradeço a Universidade Federal de Mato Grosso do Sul e ao Fundo Nacional de Desenvolvimento da Educação (FNDE).

\section{REFERÊNCIAS}

AMBROSANO, G. M. B. Modelo matemático para simulação do controle biológico de Diatraea 
saccharalis (Fabr., 1794) através de Trichogramma galloi Zucchi, 1988. 1994. Tese (Doutorado) - Universidade de São Paulo, São Paulo, SP, 1994.

BASSANEZI, R. C. Ensino-aprendizagem com modelagem matemática. 4. ed. São Paulo: Contexto, 2002.

CONAB. Acompanhamento da Safra Brasileira: Cana de açúcar. Brasília, 2021, v. 7.

DA SILVA, D. M. Modelagem Matemática Aplicada ao Controle e Manejo Integrado de Pragas em Lavouras do Milho. 2019. Dissertação (Mestrado) - Universidade Federal Fluminense, Volta Redonda, 2019.

DE CAMPOS, P. R. B. Critério de estabilidade de Routh. Curitiba: UTFPR DAELN, [20--]. Disponível em

http://paginapessoal.utfpr.edu.br/brero/controle $1 /$ material-

didatico/CRITERIO\%20DE\%20ESTABILIDADE\%20D E\%20ROUTH 02.pdf/view. Acesso em: 21 jan. 2022.

DE JESUS, I. Equações de Lotka-Volterra aplicadas ao controle biológico da broca da cana de açúcar. 2018. Dissertação (Mestrado) Universidade de Uberaba. Uberaba, MG, 2018.

GALLO, D. et al. Entomologia Agrícola. Piracicaba: FEALQ, 2002. v. 10.

MATTIUZZO, L. B. Estabilidade local dos pontos de equilíbrio em sistemas planares e hiperbólicos de EDO's de primeira ordem. Trabalho de Conclusão do Curso (Licenciatura em Matemática) - Instituto Federal de Educação, Ciência e Tecnologia de São Paulo, São Paulo, 2016.

NAVA, D. E. ; PINTO, A. de S.; SILVA, S. D. dos A. Controle biológico da broca da cana-deaçúcar. Embrapa Clima Temperado-Documentos (INFOTECA-E), 2009.

PEREIRA-BARROS, J. L. et al. Aspectos biológicos de Trichogramma galloi Zucchi, 1988 (Hymenoptera: Trichogrammatidae) criados em ovos de Diatraea saccharalis (Fabricius, 1794)(Lepidoptera: Crambidae). Ciência e Agrotecnologia, v. 29, p. 714-718, 2005.
https://doi.org/10.1590/S1413$\underline{70542005000400002}$

POLETTI, M. et al. A Broca da Diatrea Saccharalis na cultura da cana-de-açúcar e em outras culturas agrícolas no Brasil. MIP Experience, Conchal, v. 3, p. 24-26, ago., 2019. Disponível em: mip-experience-03.pdf (promip.agr.br) . Acesso em: 7 jul. 2021.

RAFIKOV, M.; LIMEIRA, E. H. Dinâmica de interação da praga da cana-de-açúcar com seu parasitóide Trichogramma galloi. In: BRAZILIAN CONFERENCE ON DYNAMICS CONTROL AND THEIR APPLICATIONS. 9., 2010. Serra Negra, SP Proceedings [...]. Serra Negra, 2010. https://doi.org/10.1080/00207160.2011.587873

RAFIKOV, M.; LIMEIRA, E. H. Mathematical modelling of the biological pest control of the sugarcane borer, International Journal of Computer Mathematics, v. 89, n. 3, 2012.

SHEIL, D.; BURSLEM, D. F. R. P; ALDER, D. The interpretation and misinterpretation of mortality rate measures. Journal of Ecology, p. 331-333, 1995. https://doi.org/10.2307/2261571 